\title{
PRECISE TRAJECTORIES AND ORBITS OF METEOROIDS FROM THE 1999 LEONID METEOR STORM
}

\author{
HANS BETLEM \\ Dutch Meteor Society, Lederkarper 4, 2318 NB Leiden, The Netherlands \\ E-mail: betlem 9 strw.leidenuniv.nl \\ PETER JENNISKENS \\ SETI Institute, NASA ARC, Mail Stop 239-4, Moffett Field, CA 94035 \\ E-mail: pjenniskens $Q$ mail.arc.nasa.gov \\ PAVEL SPURNY \\ Astronomical Institute, Ondrejov Observatory, 25165 Ondrejov, Czech \\ Republic \\ GUUS DOCTERS VAN LEEUWEN, KOEN MISKOTTE, \\ AND CASPER R. TER KUILE \\ Dutch Meteor Society, Lederkarper 4, 2318 NB Leiden, The Netherlands \\ and \\ PETER ZERUBIN AND CHRIS ANGELOS \\ Fremont Peak Observatory Association, P.O. Box 1110. San Juan Bautista, CA 95045
}

(Received 6 August 2000, Accepted 17 August 2000)

\begin{abstract}
Photographic multi-station observations of 47 Leonid meteors are presented that were obtained from two ground locations in Spain during the 1999 meteor storm. We find an unresolved compact cluster of radiants at $\alpha=153.67 \pm 0.05$ and $\delta=21.70 \pm$ 0.05 for a mean solar longitude of 235.282 ( $(2000)$. The position is identical to that of the Nov. $17 / 18$ outburst of 1998, which implies that both are due to comet 55P/TempelTuttle's ejecta from 1899 . We also find a halo which contains about $28 \%$ of all meteors. The spatial distribution of radiant positions appears to be Lorentzian, with a similar fraction of meteors in the profile wings as the meteor storm activity curve.
\end{abstract}

Keywords: Comet dust trial, dispersion, Leonids 1999, meteor, meteor orbit, meteor trajectory, orbital dynamics 
the orbits of the meteoroids to be more similar. This would be a good test for our error estimates and an invitation to push the capability of the technique to the limit. Observations of the storm were performed from Spain, while similar observations from California served for reference. Only part of the data have been reduced at this time. Here, we report on the first data measured in Spain during the 1999 Leonid meteor storm.

\section{Methods}

The measurements were made from two observing sites at Punto Alto $\left(38^{\circ} 22^{\prime} 45^{\prime \prime} .25356^{\circ} 58^{\prime} 1^{\prime \prime} .88\right.$; height $\left.772 \mathrm{~m}\right)$ and Casa Nueva $\left(39^{\circ} 07^{\prime}\right.$ $01^{\prime \prime} .30357^{\circ} 16^{\prime} 39^{\prime \prime} .90$; height $785 \mathrm{~m}$ ) in Spain. Time exposures were made by fixed cameras, while an all-sky intensified video camera recorded the time of occurrence of the bright meteors. We deployed the same clusters of small ( $35 \mathrm{~mm}$ format) cameras with $50 \mathrm{~mm}$ f/1.8 optics and crystal controlled rotating shutters as in Betlem et al. (1999). The negatives were developed, scanned on Kodak Photo $C D$, and analyzed using interactive Astroscan software in the normal manner (Betlem et al., $1997 ; 1998)$. The typical astrometric accuracy is $0.003^{\circ}$.

\section{Results}

Around 1100 meteors were photographed from Punto Alto and about 700 were photographed from Casa Nueva on the night of Nov. 17/18, 1999. The first 65 precisely reduced orbits are presented in this paper.

Of the first 65 precisely reduced orbits, 47 turn out to have convergence angles larger than 20 degrees. These provide the most accurate results and are listed in Tables I and II. The convergence angle is the angle between the two planes defined by each observing site and the meteor trajectory. Table I gives the trajectories of 47 Leonids that are part of the 1999 Leonid meteor storm. The columns list apparent visual magnitude $(\mathrm{Mv})$, beginning and end height $(\mathrm{km})$, heliocentric velocity $(\mathrm{Vh})$, geocentric velocity $(\mathrm{Vg})$, geocentric radiant coordinates $(\alpha, \delta)$, and convergence angle $(Q)$. The heights represent the lowest and highest point of the recorded trajectory, respectively, which does not take into account that some meteors may have entered the field of view, while others may have left, without their actual beginning or end point being recorded. Table II lists the corresponding orbital elements of these 47 
TABLE II

\begin{tabular}{|c|c|c|c|c|c|c|c|c|c|c|}
\hline Code & $\mathbf{q}$ & \pm 9 & $1 / a$ & $\pm 1 / 2$ & i & $\pm \mathbf{i}$ & $\omega$ & $\pm \omega$ & $\Omega$ & $\pm \Omega$ \\
\hline 1999008 & 0.9843 & 0.0002 & 0.092 & 0.022 & 162.62 & 0.07 & 172.24 & 40.23 & 235.21500 & 0.00000 \\
\hline 1999010 & 0.9844 & 0.0002 & 0.105 & 0.001 & 162.49 & 0.07 & 172.35 & 0.16 & 235.23561 & 0.00000 \\
\hline 1999011 & 0.9843 & 0.0005 & 0.098 & 0.014 & 162.65 & 0.11 & 172.28 & 0.50 & $235.2356 \mathrm{I}$ & 0.00000 \\
\hline 1999013 & 0.9842 & 0.0007 & 0.125 & 0.017 & 162.34 & 0.30 & 172.09 & 0.65 & 235.24906 & 0.00000 \\
\hline 1999014 & 0.9843 & 0.0003 & 0.043 & 0.010 & 162.06 & 0.13 & 172.38 & 0.27 & 235.25277 & 0.00000 \\
\hline 1999015 & 0.9844 & 0.0009 & 0.076 & 0.006 & 162.58 & 0.38 & 172.41 & 0.80 & 235.25314 & 0.00000 \\
\hline 1999016 & 0.9837 & 0.0005 & 0.064 & 0.012 & 162.74 & 0.18 & 171.83 & 0.39 & 235.25536 & 0.00000 \\
\hline 1999017 & 0.9845 & 0.0000 & 0.082 & 0.003 & 162.53 & 0.01 & 172.47 & 0.02 & 235.25974 & 0.00000 \\
\hline 1999018 & 0.9847 & 0.0003 & 0.137 & 0.013 & 162.47 & 0.15 & 172.56 & 0.32 & 235.25992 & 0.00000 \\
\hline 1999026 & 0.9850 & 0.0005 & 0.105 & 0.012 & 162.73 & 0.23 & 172.93 & 0.49 & 235.26866 & 0.00000 \\
\hline 1999028 & 0.9845 & 0.0003 & 0.078 & 0.013 & 162.42 & 0.11 & 172.51 & 0.23 & 235.27117 & 0.00000 \\
\hline 1999029 & 0.9842 & 0.0005 & 0.103 & 0.021 & 162.51 & 0.22 & 172.11 & 0.49 & 235.27140 & 0.00000 \\
\hline 1999030 & 0.9841 & 0.0002 & 0.071 & 0.008 & 162.53 & 0.11 & 172.16 & 0.20 & 235.27218 & 0.00000 \\
\hline 1999031 & 0.9863 & 0.0004 & 0.123 & 0.045 & 161.76 & 0.24 & 174.22 & 0.52 & 235.27277 & 0.00000 \\
\hline 1999032 & 0.9846 & 0.0046 & 0.078 & 0.005 & 162.64 & 0.02 & 172.56 & 0.07 & 913 & 0.00000 \\
\hline 1999033 & 0.9843 & 0.0002 & 0.092 & 0.022 & 162.62 & 0.07 & 172.24 & 0.23 & 235.21500 & 0.00000 \\
\hline 1999036 & 0.9846 & 0.0002 & 0.107 & 0.006 & 162.54 & 0.06 & 172.49 & 0.21 & 235.27618 & 0.00000 \\
\hline 1999037 & 0.9839 & 0.0003 & 0.134 & 0.023 & 162.39 & 0.12 & 171.85 & 0.28 & 235.27697 & 0.00000 \\
\hline 1999041 & 0.9839 & 0.0005 & 0.092 & 0.022 & 162.86 & 0.21 & 171.95 & 0.45 & 235.27856 & 0.00000 \\
\hline 1999043 & 0.9848 & 0.0000 & 0.115 & 0.117 & 162.51 & 0.01 & 172.69 & 0.04 & 235.28078 & 0.00000 \\
\hline 1999044 & 0.9845 & 0.0001 & 0.102 & 0.005 & 162.51 & 0.05 & 172.40 & 0.11 & 235.28111 & 0.00000 \\
\hline 1999045 & 0.9829 & 0.0005 & 0:093 & 0.011 & 162.50 & 0.20 & 171.08 & 0.43 & 235.28119 & 0.00000 \\
\hline 1999046 & 0.9852 & 0.0007 & 0.100 & 0.026 & 162.97 & 0.36 & 173.16 & 0.77 & 235.28150 & 0.00000 \\
\hline 1999047 & 0.9874 & 0.0002 & 0.105 & 0.007 & 162.93 & 0.19 & 175.93 & 0.40 & 23 & 0.00000 \\
\hline 1999050 & 0.9844 & 0.0000 & 0.082 & 0.010 & 162.49 & 0.02 & 172.34 & 0.06 & 235. & 0.00000 \\
\hline 1999057 & 0.9845 & 0.0002 & 0.077 & 0.030 & 162.65 & 0.07 & 172.52 & 0.18 & 235. & 0.00000 \\
\hline 1999058 & 0.9846 & 0.0000 & 0.078 & 0.011 & 162.38 & 0.02 & 172.56 & 0.06 & 235.28807 & 0.00000 \\
\hline 1999059 & 0.9845 & 0.0002 & 0.100 & 0.010 & 162.56 & 0.06 & 172.40 & 0.14 & 235.29373 & 0.00000 \\
\hline 1999061 & 0.9850 & 0.0003 & 0.086 & 0.013 & 162.66 & 0.13 & 172.91 & 0.27 & 235.28957 & 0.00000 \\
\hline 1999064 & 0.9817 & 0.0000 & 0.095 & 0.004 & 162.08 & 0.01 & 170.16 & 0.04 & 235.29085 & 0.00000 \\
\hline 1999065 & 0.9843 & 0.0003 & 0.097 & 0.018 & 162.58 & 0.13 & 172.27 & 0.29 & 235.58202 & 0.00000 \\
\hline 1999066 & 0.9857 & 0.0003 & 0.165 & 0.017 & 162.20 & 0.10 & 173.53 & 0.37 & 235.29116 & 0.00000 \\
\hline 1999067 & 0.9838 & 0.0004 & 0.122 & 0.008 & 162.25 & 0.17 & 171.78 & 0.37 & 235.29149 & 0.00000 \\
\hline | 999071 & 0.9833 & 0.0003 & 0.151 & 0.016 & 162.31 & 0.09 & 171.22 & 0.29 & 235.29283 & 0.00000 \\
\hline 1999074 & 0.9844 & 0.0002 & 0.124 & 0.014 & 162.46 & 0.08 & 172.27 & 0.17 & 235.29479 & 0.00000 \\
\hline 1999079 & 0.9833 & 0.0007 & 0.119 & 0.011 & 162.47 & 0.27 & 171.36 & 0.58 & 235.29797 & 0.00000 \\
\hline 1999081 & 0.9848 & 0.0008 & 0.089 & 0.015 & 162.61 & 0.35 & 172.74 & 0.74 & 235.29842 & 0.00000 \\
\hline & 0.9842 & 0.0001 & 0.106 & 0.009 & 162.47 & 0.02 & 172.15 & 0.07 & 235.30104 & 0.00000 \\
\hline 2087 & 0.9844 & 0.0003 & 0.072 & 0.025 & 162.42 & 0.15 & 172.41 & 0.32 & 235.29975 & 0.00000 \\
\hline & 0.9843 & 0.0001 & 0.079 & 0.002 & 162.65 & 0.03 & 172.26 & 0.06 & 235.30348 & 0.00000 \\
\hline 1999105 & 0.9857 & 0.0015 & 0.062 & 0.023 & 162.37 & 0.79 & 173.75 & 1.65 & 235.31298 & 0.00001 \\
\hline 1999106 & & 0.0001 & 0.116 & 0.006 & 162.52 & 0.04 & 172.22 & 0.13 & 235.31273 & 0.00000 \\
\hline 1999112 & 0.9828 & 0.0005 & 0.084 & 0.029 & 162.26 & 0.13 & 171.06 & 0.41 & 235.30944 & 0.00000 \\
\hline 1999114 & 0.9838 & 0.0008 & 0.122 & 0.012 & 162.37 & 0.16 & 171.80 & 0.69 & 235.32150 & 0.00000 \\
\hline 1999115 & 0.9843 & 0.0003 & 0.129 & 0.007 & 162.65 & 0.13 & 172.14 & 0.27 & 235.32358 & 0.00000 \\
\hline 1999117 & 0.9851 & 0.0001 & 0.083 & 0.006 & 162.67 & 0.03 & 173.10 & 0.11 & 235.32502 & 0.00000 \\
\hline 1999129 & 0.9844 & 0.0002 & 0.095 & 0.004 & 162.59 & 0.06 & 172.36 & 0.17 & 235.34490 & 0.00000 \\
\hline & .9844 & & $\begin{array}{l}0.099 \\
0.024\end{array}$ & 6 & $\begin{array}{l}162.50 \\
0.22\end{array}$ & 0.14 & $\begin{array}{l}172.3 \\
0.86\end{array}$ & & & \\
\hline
\end{tabular}


-0.36 degrees per degree solar longitude. In this system, the 1999 Leonid storm radiant was at $\alpha=153.39 \pm 0.05$ and $\delta=21.80 \pm 0.05$ (J2000). Compare this to the radiant position of the 1998 outburst on Nov, 17/18, at $\alpha=153.43 \pm 0.09$ and $\delta=21.97 \pm 0.14$ (Betlem et al., 1999). We conclude that both are identical, which implies that the second outburst in 1998 was in fact caused by the same dust trailet responsible for the 1999 Leonid storm. Based on the models by McNaught and Asher (1999), both must be due to ejecta from 1899.

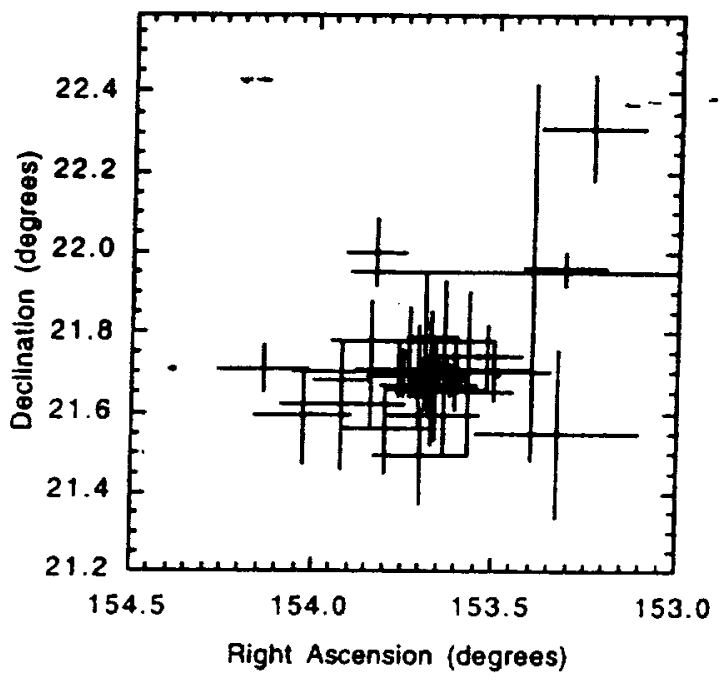

Figure 2. Geocentric radiant position of Leonids at the time of the 1999 Leonid storm.

With at least 13 out of 47 meteors ( $28 \%$ ) part of this halo, it is unlikely that the halo is due to the relatively weak annual Leonid shower or the (almost) absent Leonid Filament (Jenniskens and Betlem, 2000). The halo implies strong wings in the radiant dispersion of the storm, unlike a Gaussian distribution. Instead, the spatial radiant distribution appears similar to the Lorentzian shape of the nodal distribution (Jenniskens $\boldsymbol{e t}$ al., 2000). Indeed, the relative flux in the background profile versus the total flux curve of that Lorentzian curve is $28 \%$, which is in agreement with the distribution of radiant positions.

The distribution of orbital elements may serve to improve meteor stream models. It is very significant that all observed orbits are well dated, being ejected during the return of comet 55P/Tempel-Tuttle in 